\title{
Estados de Humor e Resultado Esportivo de Uma Equipe ao Longo da Segunda Fase da Liga Nacional de Futsal
}

\author{
Guilherme Guimarães Bevilacqua $^{1}$ (D), Maick da Silveira Viana ${ }^{2}$ (D), \\ Paulo José Barbosa Gutierres Filho ${ }^{3}$ (D), Vitor da Silveira Borges ${ }^{1}$ (D), \& Ricardo Brandt ${ }^{4,}$ * (DD \\ ${ }^{1}$ Universidade do Estado de Santa Catarina, Florianópolis, SC, Brasil \\ ${ }^{2}$ Instituto Federal de Santa Catarina, Joinville, SC, Brasil \\ ${ }^{3}$ Universidade de Brasília, Brasília, DF, Brasil \\ ${ }^{4}$ Universidade Estadual do Oeste do Paraná, Marechal Cândido Rondon, PR, Brasil
}

\begin{abstract}
RESUMO - O objetivo do estudo foi avaliar o humor de atletas profissionais de futsal durante a segunda fase da Liga Futsal Brasileira 2012. A amostra foi composta por 15 atletas do sexo masculino e o instrumento utilizado para avaliação foi a Escala de Humor de Brunel. Os resultados mostram que houve diferenças significativas $(\mathrm{p}<0,05)$ dos estados de humor no dia anterior, pré-jogo e pós-jogo. A equipe apresentou estados de humor correspondente ao perfil iceberg em quase todos os momentos: vigor constante e elevado, com exceção do pós-jogo nas derrotas; e fadiga com índices baixos, que se apresentaram elevadas no pós-jogo. Conclui-se que os estados de humor podem intervir positivamente ou negativamente no desempenho esportivo da equipe.
\end{abstract}

PALAVRAS-CHAVE: estados de humor, atletas, futsal

\section{Mood States and Sports Results of a Team Throughout the Second Phase of the National Futsal League}

\begin{abstract}
This study evaluated the moods states of professional futsal players during the second phase of the Brazilian Futsal League 2012. The sample was composed of 15 male athletes and the instrument used for evaluation was the Brunel Mood Scale. The results show that there were significant differences $(p<0.05)$ in mood states on the previous day, pregame and post-game. The team presented mood states corresponding to the iceberg profile in almost all times: constant and high vigor, except for the post-game defeats, and fatigue with low levels, which were elevated in the post-game. It was concluded that mood states can positively or negatively interfere with team sports performance.
\end{abstract}

KEYWORDS: mood states, athletes, indoor soccer

$\mathrm{Na}$ última década, houve um aumento no número de estudos de Psicologia do Esporte que se concentraram na compreensão da relação entre emoções e o desempenho dos atletas durante a competição (Andrade, Bevilacqua, Coimbra, Pereira, \& Brandt, 2016).

Atletas de elite fazem sacrifícios consideráveis na busca da melhor condição física, técnica, tática e psicológica (Beedie, Terry, \& Lane, 2000; Brandt, Bevilacqua, \& Andrade, 2016). Neste sentido, diversos estudos buscam compreender a importância do estado emocional no desempenho esportivo, pois consideram haver relação entre os fatores psicológicos e o esporte (Brandt, Bevilacqua, \& Andrade, 2017; Brandt et al., 2011; Devenport, Lane, \& Hanin, 2005; Vieira, Fernandes, Vieira, \& Vissoci, 2008; Werneck, Coelho, \& Ribeiro, 2002). Embora o desempenho esportivo seja considerado um constructo multifatorial, historicamente tem sido investigado a partir da perspectiva de disciplinas únicas das ciências do esporte, tais como Fisiologia do Esporte, Biomecânica do Esporte e Psicologia do Esporte (Glazier, 2017).

\footnotetext{
*E-mail: ricabrandt@gmail.com

- Submetido: 27/07/2016; Revisado: 16/02/2018; Aceito: 26/03/2018.
} 
Nos últimos anos, os fatores psicológicos relacionados ao desempenho esportivo receberam atenção especial e percebeu-se que os estados de humor abarcam uma parcela significativa dos estudos que buscam compreender esse fenômeno (Brandt et al., 2016). Os estados de humor podem influenciar, positiva ou negativamente, o resultado final de uma partida ou campeonato, determinando o sucesso ou o fracasso na busca dos objetivos almejados (Araujo \& Gomes, 2005; Brandt et al., 2011; Devenport et al., 2005; Vieira et al., 2008). O humor foi operacionalizado e medido como um construto composto de seis estados: tensão, depressão, raiva, vigor, fadiga e confusão (Lane \& Terry, 2000).

Estudos anteriores mostram que os estados de humor são preditivos do desempenho (Andrade et al., 2016). Na busca por melhores resultados, o esportista de alto rendimento vive situações constantes de estresse físico e mental e deve saber lidar com as emoções relacionadas ao sucesso e ao fracasso, exigindo maior controle de seus estados de humor. Estudos demonstram que o humor do atleta é diretamente influenciado pelo resultado do jogo, de modo que os estados de humor após as partidas são significativamente diferentes entre vitórias e derrotas (Andrade et al., 2016; Brandt et al., 2016; dos Santos, 2008; Vieira et al., 2008).

No futsal há grande exigência psico e neuromotora dos atletas, caracterizado por disputas de alta intensidade e elevada demanda energética. Entretanto, o nível de exigência das partidas depende do tipo de competição disputada, da posição em quadra, do padrão de jogo da equipe, das condições táticas e técnicas, e das dimensões da quadra. Entre os fatores que influenciam o desempenho dos atletas durante as competições estão o curto intervalo de recuperação entre as partidas, devido ao calendário extenuante, a composição corporal e os estados de humor (Cyrino, Altimari, Okano, \& de Faria Coelho 2002.; de Rose
Junior, 2002; Garcia, 2004; Soares \& Tourinho Filho, 2006). O desempenho do atleta está relacionado com o seu humor e o indivíduo que demonstra maior nível de vigor, animação e alegria tende enfrentar uma situação frustrante com mais coragem, diferente daquele com maior nível de depressão ou desesperança, o qual tenderá a apresentar comportamento de desistência ou fuga de determinada situação (Brandt et al., 2011; dos Santos, 2008; Vieira et al., 2008).

De acordo com a literatura, de maneira geral, os atletas que apresentam vigor mais elevado em comparação às variáveis negativas do humor (tensão, depressão, raiva, fadiga e confusão mental) possuem um perfil de humor conhecido como perfil iceberg (Lane \& Terry, 2000). O oposto, ou seja, elevada tensão, depressão, raiva, fadiga e confusão mental, associadas ao baixo vigor, pode ser considerado como humor deprimido (Lane \& Terry, 2000). De modo geral, quanto mais próximo ao perfil iceberg, melhores são as chances de um bom rendimento do atleta (Brandt et al., 2010; Brandt et al., 2016).

Os atletas em período competitivo experimentam diferentes aspectos emocionais (níveis elevados de estresse, tomada de decisão, alterações no padrão de sono e modificações do humor) (Fullagar et al., 2015; Furley, Bertrams, Englert, \& Delphia, 2013; Harrison \& Home, 2000; Souissi et al., 2013), os quais podem afetar seu desempenho nas competições. Apesar disso, não foram encontrados estudos publicados, nacional $\mathrm{e}$ internacionalmente, sobre alterações do humor e suas possíveis relações com os resultados dos jogos de atletas de alto rendimento de futsal. Tendo em vista esse contexto, o propósito do presente estudo foi avaliar os estados de humor de atletas de futsal profissional de uma equipe do Sul do Brasil durante a segunda fase da Liga Futsal Brasileira 2012.

\section{MÉTODO}

\section{Participantes}

Os participantes, atletas de uma equipe que disputou a Liga Futsal 2012, foram selecionados de maneira não aleatória. Alguns atletas estiveram fora da coleta em determinadas partidas por estarem suspensos ou lesionados. Portanto, os dados foram coletados entre os atletas que compunham a equipe em cada jogo.

O estudo foi realizado com 15 atletas durante toda a segunda fase da Liga Futsal, classificatória para as quartas-de-final. A média de idade da equipe foi de 28 anos $(\mathrm{DP}=5,32)$ [mínimo 19 anos, máximo 36 anos]; a estatura média foi de $1,76 \mathrm{~m}(\mathrm{DP}=6,20)$ [mínima $1,65 \mathrm{~m}$, máxima $1,87 \mathrm{~m}$ ]; o peso médio foi de $76 \mathrm{~kg}(\mathrm{DP}=7,17)$ [mínimo $63 \mathrm{~kg}$, máximo $92 \mathrm{~kg}$ ]. A idade, peso e altura dos atletas participantes da pesquisa foram separados pela posição em que atuavam. A maioria dos atletas concluiu $(93,4 \%)$ o Ensino Médio; quatro $(26,6 \%)$ recebiam patrocínios, sendo que dois $(13,3 \%)$ contavam com remuneração financeira e outros dois (13,3\%), com outras formas de auxílio.

Em 2012, durante a primeira fase, a competição contou com 20 equipes participantes que jogaram entre si, num modelo de rodízio simples em grupo único. Classificaram-se para a $2^{\mathrm{a}}$ fase as 16 equipes mais bem colocadas na tabela. $\mathrm{Na}$ segunda fase, foco deste estudo, as equipes classificadas foram distribuídas equitativamente em quatro grupos (A, $\mathrm{B}, \mathrm{C}, \mathrm{D}$ ) que jogaram entre si, em rodízio duplo (turno e returno). Os grupos foram formados, em todas as fases, seguindo critérios da Confederação Brasileira de Futsal. As duas melhores equipes de cada chave disputaram as quartas-de-final ( $3^{\text {a }}$ fase) no modelo "mata mata", assim como a semifinal ( $4^{\mathrm{a}}$ fase $)$ e a final. 


\section{Instrumentos}

Foram utilizados dois instrumentos, descritos a seguir.

O Questionário de Caracterização foi utilizado com o objetivo de obter uma caracterização geral dos atletas (Brandt, 2008). Esse instrumento era composto por questões abertas e fechadas para a verificação de idade, estatura, peso, escolaridade, posição em que atua na equipe e existência ou não de patrocínio.

A Escala de Humor de Brunel - BRUMS (Terry, Lane, \& Fogarty, 2003), validada para o Brasil por Rohlfs (2006), visava avaliar os estados de humor dos atletas. Em sua validação nacional, a Brums apresentou boa consistência interna, com valores de alfa de Cronbach superiores a 0,70, sendo considerada, dessa forma, um instrumento confiável para mensurar os estados de humor de atletas brasileiros (Rohlfs, 2006; Rohlfs et al., 2008). Essa escala apresenta 24 itens, com respostas correspondentes a cinco níveis $(0=$ nada/4=extremamente), devendo o atleta responder como está se sentindo no momento da coleta. Os 24 itens da escala são agrupados em seis subescalas (tensão, depressão, raiva, vigor, fadiga e confusão mental), cada uma composta por quatro itens. A soma das respostas de cada subescala resulta num escore que varia de 0 a 16 pontos.

\section{Procedimento}

A aprovação da pesquisa foi realizada pelo Comitê de Ética em Pesquisa em Seres Humanos da Universidade do Estado de Santa Catarina (protocolo $n^{\circ} 33 / 2007$ ). Foram explicados aos atletas participantes os objetivos da pesquisa, assim como os procedimentos e a forma de preenchimento dos questionários. Os atletas foram orientados a expressar a verdade nas respostas do instrumento com a garantia de que as informações fornecidas e sua identidade seriam sigilosas. Todos assinaram o Termo de Consentimento Livre e Esclarecido anteriormente à coleta de dados.

A coleta de dados foi realizada entre os meses de junho e agosto de 2012, durante a segunda fase da Liga Futsal 2012, e contou com a colaboração de um membro da comissão técnica da equipe. A aplicação do Brums ocorreu em três momentos diferentes de cada jogo: dia anterior ao jogo, pré-jogo e pós-jogo, num total de seis jogos. Procurou-se realizar a aplicação do instrumento até $1 \mathrm{~h}$ antes (pré) e 1 $h$ depois (pós) de cada partida. No dia anterior aos jogos, os questionários foram aplicados antes do último treino que precedia o jogo. O questionário pré-jogo foi aplicado quando os jogadores se concentravam para as partidas no vestiário. O questionário pós-jogo, por sua vez, foi aplicado entre a saída do vestiário e a chegada ao hotel em que ficaram hospedados, quando os jogos foram fora da cidade em que o clube se localizava. Nos jogos realizados na cidade dos atletas, os questionários foram respondidos no vestiário após o jogo.

\section{Análise de Dados}

Os dados foram analisados por meio do programa Statistical Package for Social Science (SPSS) versão 20,0. Para o tratamento dos dados foram utilizadas a estatística descritiva (frequência, porcentagem, média e desvio padrão) e a estatística inferencial (teste de Shapiro-Wilk). Nesta última estatística, levando em conta que parte dos dados não se distribuiu normalmente (tensão, depressão, raiva e confusão mental), o perfil de humor dos atletas foi comparado por meio do teste de Wilcoxon, e as demais variáveis (vigor, fadiga), por meio do teste $\mathrm{T}$ pareado. $\mathrm{O} \alpha$ estabelecido foi de $0,05(p<0,05)$.

\section{RESULTADOS}

Durante a segunda fase da Liga Futsal 2012, foco do presente estudo, das seis partidas disputadas, a equipe venceu três, empatou uma e perdeu duas. A Tabela 1 apresenta os valores médios dos estados de humor dos atletas investigados em cada partida.

De modo geral, nos dias anteriores aos jogos, os atletas apresentaram baixa tensão. A tensão aumentou significativamente $(p<0,05)$ do dia anterior para o préjogo, nas partidas em que não venceram (jogos $2,4 \mathrm{e}$ 6 - símbolo * da coluna tensão na Tabela 1) e diminuiu significativamente quando comparados os pré-jogos com pós-jogos nas partidas 1, 2, 4 e 5 (símbolo $\dagger$ da coluna tensão na Tabela 1), evidenciando não haver relação entre tensão e o resultado do jogo. Diferenças significativas entre o dia anterior e o pós-jogo foram encontradas apenas nas partidas finais (jogos 5 e 6), aquelas que definiram a classificação da chave. Na comparação entre os jogos, a tensão no dia anterior à partida 2 diminuiu significativamente em relação ao dia anterior à partida 1 , e houve aumento estatisticamente significativo entre os pré-jogos e pós-jogos das partidas 5 e 6 .

Nos jogos em que a equipe foi derrotada (2 e 6), houve aumento significativo da depressão na comparação do préjogo com o pós-jogo e na comparação do dia anterior com pós-jogo. Entre os jogos houve aumento significativo no pós-jogo das partidas em que houve derrota, em comparação com o pós-jogo da partida anterior ao resultado negativo (1 e 5) e diminuição significativa comparando a partida 2 com a partida 3 .

Diferenças significativas para a raiva foram encontradas nas partidas em que a equipe perdeu, onde houve grande aumento pós-jogo deste estado de humor em relação ao dia anterior e pré-jogo. A raiva encontrada pós-jogo nas partidas em que houve derrota ( 2 e 6 ) também teve aumento significativo comparando à raiva pós-jogo das partidas anteriores (1 e 5). 
Tabela 1

Estados de humor de jogadores de futsal no dia anterior, pré e pós-jogos durante a segunda fase da Liga Futsal 2012

\begin{tabular}{|c|c|c|c|c|c|c|c|}
\hline & & Tensão & Depressão & Raiva & Vigor & Fadiga & Confusão Mental \\
\hline \multirow{3}{*}{$\underset{\square}{\Sigma}$} & DA & $¥ 2,17 \pm 2,69$ & $0,75 \pm 1,35$ & $2,17 \pm 2,75$ & $¥ 11,0 \pm 3,71^{\#}$ & $2,58 \pm 1,78^{* \#}$ & $1,08 \pm 1,5$ \\
\hline & Pré & $2,75 \pm 1,91^{\dagger}$ & $0,58 \pm 1,73$ & $0,92 \pm 1,67$ & $12,0 \pm 4,86$ & $0,17 \pm 0,57^{* \dagger}$ & $0,50 \pm 1,24$ \\
\hline & Pós & $0,50 \pm 1,44^{\dagger}$ & ${ }^{£} 0 \pm 0$ & ${ }^{£} 1,0 \pm 2,48$ & ${ }^{£} 8,50 \pm 2,84^{\#}$ & $6,0 \pm 4,17^{\# \dagger}$ & $0,17 \pm 0,57$ \\
\hline \multirow{3}{*}{$\underset{\sim}{\stackrel{(}{\ominus}}$} & DA & ${ }^{\ddagger} 0,92 \pm 1,37^{*}$ & $0,33 \pm 0,65^{\#}$ & $1,50 \pm 2,31^{\#}$ & э¥ $7,42 \pm 4,77^{\#}$ & $3,92 \pm 2,35^{*}$ & $0,42 \pm 1,16$ \\
\hline & Pré & $2,50 \pm 2,31^{* \dagger}$ & $0,33 \pm 0,88^{\dagger}$ & $0,42 \pm 0,99^{\dagger}$ & $12,33 \pm 3,49^{\dagger}$ & $0,75 \pm 1,21^{* \dagger}$ & $0,58 \pm 1,24$ \\
\hline & Pós & $1,08 \pm 1,5^{\dagger}$ & $£ ५ 4,75 \pm 3,49^{\# \dagger}$ & ${ }^{ \pm} 6,5 \pm 3,96^{\# \dagger}$ & єи $3,92 \pm 3,98^{\# \dagger}$ & $5,0 \pm 3,27^{\dagger}$ & $0,75 \pm 1,35$ \\
\hline \multirow{3}{*}{$\underset{n}{\sum}$} & DA & $1,67 \pm 1,72$ & $0,58 \pm 1,37$ & $1,08 \pm 1,97$ & ${ }^{\ni} 11,25 \pm 2,95^{\#}$ & ${ }^{\ni} 1,17 \pm 1,26^{\#}$ & $1,08 \pm 1,97$ \\
\hline & Pré & $1,67 \pm 2,01$ & $0,42 \pm 1,16$ & $0,83 \pm 1,99$ & ${ }^{\kappa} 10,92 \pm 3,72^{\dagger}$ & $1,08 \pm 1,62^{\dagger}$ & $0,75 \pm 1,76$ \\
\hline & Pós & $1,42 \pm 3,82$ & $1,33 \pm 4,61$ & $2,67 \pm 6,22$ & $8,58 \pm 3,14^{\# \dagger}$ & $4,75 \pm 2,8^{\# \dagger}$ & $1,17 \pm 3,73$ \\
\hline \multirow{3}{*}{ 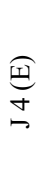 } & DA & $1,36 \pm 1,74^{*}$ & $0,18 \pm 0,4$ & $0,55 \pm 1,21$ & $10,18 \pm 4,42^{*}$ & $1,0 \pm 1,48^{* \#}$ & $0,27 \pm 0,64$ \\
\hline & Pré & $2,82 \pm 2,04^{* \dagger}$ & $0,18 \pm 0,40$ & $0,82 \pm 1,4$ & ${ }^{\kappa} 13,55 \pm 2,54^{* \dagger}$ & $0,18 \pm 0,6^{* \dagger}$ & $0,36 \pm 0,92$ \\
\hline & Pós & $0,45 \pm 0,82^{\dagger}$ & $0,36 \pm 0,8$ & $0,27 \pm 0,64$ & $7,27 \pm 4,58^{\dagger}$ & $5,64 \pm 4,5^{\# \dagger}$ & $0 \pm 0$ \\
\hline \multirow{3}{*}{$\sum_{n}^{2}$} & DA & $1,82 \pm 1,4^{\#}$ & $0,09 \pm 0,3$ & $0,18 \pm 0,6$ & ${ }^{\circledR} 11,27 \pm 3,43^{* \#}$ & $2,0 \pm 1,89^{* \#}$ & $0,27 \pm 0,9$ \\
\hline & Pré & ${ }^{€} 2,0 \pm 1,75^{\dagger}$ & $0,25 \pm 0,62$ & $0,42 \pm 0,99$ & $13,17 \pm 2,51^{* \dagger}$ & $0,33 \pm 0,88^{* \dagger}$ & $0,17 \pm 0,57$ \\
\hline & Pós & ${ }^{\#} 0,58 \pm 0,79^{\# \dagger}$ & ${ }^{*} 0,33 \pm 0,88$ & ${ }^{*} 1,25 \pm 4,33$ & \#8,67 $\pm 3,17^{\# \dagger}$ & $7,08 \pm 3,39^{\# \dagger}$ & ${ }^{*} 0,17 \pm 0,57$ \\
\hline \multirow{3}{*}{$\underset{b}{\bigoplus}$} & DA & $1,0 \pm 1,54^{* \#}$ & $0,27 \pm 0,9^{\#}$ & $0,18 \pm 0,6^{\#}$ & ${ }^{\oplus 9,45 \pm 4,8^{* \#}}$ & $3,73 \pm 3,19^{* \#}$ & $0,36 \pm 1,2^{\#}$ \\
\hline & Pré & ${ }^{€} 3,09 \pm 1,81^{*}$ & $0,27 \pm 0,9^{\dagger}$ & $\begin{array}{c}1,64 \pm 3,74^{\dagger} \\
3,74^{\dagger}\end{array}$ & $12,73 \pm 2,72^{* \dagger}$ & $0,55 \pm 1,21^{* \dagger}$ & $0,27 \pm 0,9^{\dagger}$ \\
\hline & Pós & $\# 4,27 \pm 2,41^{\#}$ & \# $12,18 \pm 4,70^{\# \dagger}$ & W $12,45 \pm 4,45^{\text {\#† }}$ & ${ }^{*} 2,18 \pm 2,82^{\# \dagger}$ & $6,18 \pm 3,84^{\# \dagger}$ & ${ }^{\#} 2,91 \pm 3,08^{\# \dagger}$ \\
\hline
\end{tabular}

Nota: Jogo (J), Vitória (V), Empate (E), Derrota (D), Dia Anterior (DA).

Símbolos iguais à direita representam variação significativa $(\mathrm{p}<0,05)$ intrajogos.

Símbolos iguais à esquerda representam variação significativa $(\mathrm{p}<0,05)$ interjogos.

Intrajogos: *: DA-Pré; Pré-Pós: †; Pós-DA: \#

Interjogos: DA. ¥: Jogo1-Jogo2; ৩: Jogo2-Jogo3; Ф: Jogo5-Jogo6

Interjogos: Pré. א: Jogo3-Jogo4; €: Jogo5-Jogo6

Interjogos: Pós. £: Jogo1-Jogo2; ų: Jogo2-Jogo3; \#ogo5-Jogo6

Com exceção dos valores encontrados para vigor no pós-jogo das partidas em que houve derrota (jogos 2 e 6), em todas as outras as médias se mostraram elevadas. Houve diminuição estatisticamente significativa do dia anterior para o pré-jogo na partida 4, 5 e 6 . Comparando o pré-jogo com o pós-jogo, foi encontrado aumento significativo do vigor nos jogos 2, 3, 4, 5 e 6 .

Na comparação do vigor encontrado no dia anterior com o encontrado no pós-jogo, houve diminuição estatisticamente significativa nos jogos 1, 2, 5 e 6; na mesma comparação, foi encontrado aumento estatisticamente significativo apenas na partida 3. Quando a comparação é feita entre os jogos para o fator vigor no dia anterior, encontrou-se aumento estatisticamente significativo do jogo 2 para o jogo 3 . Houve diminuição significativa, também no dia anterior, comparando os jogos 1 e 2,5 e 6 . No pré-jogo, houve aumento significativo apenas na comparação entre os jogos 3 e 4 . Nos pós-jogos, houve diminuição estatisticamente significativa entre os jogos 1 e 2 , e 5 e 6 e aumento significativo entre os jogos 2 e 3 .

Em todas as partidas também foram encontrados valores elevados para fadiga no pós-jogo. Houve diminuição estatisticamente significativa para fadiga comparando o dia anterior com o pré-jogo nas partidas 1, 2, 4, 5 e 6 . Verificou-se aumento estatisticamente significativo do dia anterior com o pós-jogo nas partidas 1, 3, 4, 5 e 6 . Em todos os jogos ocorreu aumento estatisticamente significativo da fadiga comparando o pré-jogo com o pós-jogo. Apenas entre as partidas $2 \mathrm{e}$ 3 houve uma diminuição estatisticamente significativa, comparando a fadiga no dia anterior entre os dois jogos.

Não foram encontrados valores elevados para confusão mental. Encontrou-se aumento estatisticamente significativo comparando o dia anterior com o pós-jogo, e comparando o pré-jogo com o pós-jogo, apenas na última partida. Entre as partidas, houve um aumento estatisticamente significativa entre o pós-jogo das partidas 5 e 6 . 


\section{DISCUSSÃO}

O propósito do presente estudo foi avaliar os estados de humor de atletas de futsal profissional de uma equipe do Sul do Brasil durante a segunda fase da Liga Futsal Brasileira 2012.

Em muitos esportes coletivos, devido à brevidade do calendário esportivo, as competições são realizadas num curto prazo e com poucos dias de intervalo entre as partidas, o que pode influenciar nos estados de humor dos atletas. No desporto de alto nível, as emoções do atleta podem sofrer diversas influências providas das situações vividas, afetando seu desempenho positiva ou negativamente, dependendo da exigência do desporto em questão e da forma como o atleta externa suas emoções (Brandt et al., 2016; Brandt et al., 2010; Lazarus, 2000).

Neste estudo houve variação dos estados de humor nas comparações dos diferentes momentos dos jogos, influenciados por fatores como importância da partida e consequente pressão pelo resultado positivo. Os achados deste estudo são consistentes com os encontrados por outros autores que sugerem haver variação dos estados de humor entre as partidas (Brandt et al., 2016; Cyrino et al., 2002; de Rose Junior, 2002; Lane, Terry, Beedie, Curry, \& Clark, 2001; Lowter \& Lane, 2002; Vieira et al., 2008).

O fator tensão descreve a tensão músculo esquelética que, em alguns momentos, pode ser observada por manifestações psicomotoras como agitação e inquietação (Viana, Almeida \& Santos, 2001; Terry, 1995). Com exceção do último jogo, no qual houve aumento da tensão pós-jogo, todos os outros valores encontrados foram superiores no pré-jogo, já que este é naturalmente um período de maior apreensão. No esporte de alto nível é constante a busca e cobrança pelo resultado positivo, mas quando o objetivo traçado não é alcançado, observa-se o aumento da tensão e o descontrole emocional do atleta, causando insegurança, visto que o treinamento não é garantia de resultado positivo nas partidas (Cratty, 1984; de Rose Junior, 2002; dos Santos, 2008;).

A depressão, aqui, é indicativa de humor deprimido e não depressão clínica. Representa sentimentos como desânimo, tristeza, infelicidade e solidão (Lane, et al., 2001; Rohlfs et al., 2008; Viana et al., 2001). De acordo com a literatura, este pode ser um fator indicativo do descontentamento da equipe diante do resultado obtido, observado na Tabela 1 pelos altos valores encontrados na segunda e sexta partida, sendo que nesta última ocorreu a eliminação da equipe na competição (Brandt et al., 2011; dos Santos, 2008).

A raiva corresponde a sentimentos de mau humor e hostilidade em relação aos outros e a si mesmo (Rohlfs et al., 2008; Spielberger, 1991; Viana et al., 2001). Valores elevados para raiva foram encontrados ao final das partidas em que a equipe foi derrotada. De acordo com Rohlfs et al. (2008), alterações negativas na raiva são influenciadas por resultados adversos.

$\mathrm{O}$ fator vigor representa um estado de energia e vigor físico (Rohlfs et al., 2008; Spielberger, 1991; Viana et al., 2001). Com exceção do pós-jogo nas derrotas, a equipe investigada apresentou perfil de estado de humor com valores altos para vigor nos seis jogos, demonstrando placares sem discrepâncias e com consequente equilíbrio nas disputas. Estudos sugerem que valores altos para vigor refletem num bom desempenho esportivo (Vieira et al., 2008). Por se tratar de um esporte com grande exigência física e neuromotora, fisiologicamente já era esperado a diminuição do vigor e aumento da fadiga pós-jogo, mas essa diminuição pode ser acentuada devido às emoções atreladas à derrota (dos Santos, 2008).

Quanto à fadiga, caracterizada por cansaço e baixa energia (Viana et al., 2001), foram encontradas diferenças significativas em quase todas as partidas comparando o pósjogo com outros momentos. Diversos autores evidenciam o desgaste físico pós-competitivo, caracterizado neste estudo pelo aumento da fadiga (Brandt et al., 2011; Rohlfs et al., 2008). Independentemente do resultado, observa-se um aumento significativo da fadiga no pós-jogo em todos os jogos. Esse elevado índice pode ser explicado pela demanda energética e exigência psico e neuromotora do futsal (Cyrino et al., 2002; Garcia, 2004; de Rose Junior, 2002; Soares \& Tourinho Filho, 2006).

A confusão mental é caracterizada por um estado de confusão e baixa lucidez, em que o atleta pode apresentarse inseguro e confuso (Viana et al., 2001). Neste estudo, a confusão mental apresentou-se dentro do esperado em praticamente todos os momentos. Essa elevação na confusão mental do último jogo evidencia a insegurança dos atletas perante o resultado adverso e consequente desclassificação da equipe.

\section{CONSIDERAÇÕES FINAIS}

No presente estudo foram encontradas diferenças estatisticamente significativas em diferentes momentos, ao longo da competição, nos estados de humor dos atletas participantes desta pesquisa. Níveis elevados de tensão, depressão, raiva, fadiga e confusão mental foram observados, principalmente no pré-jogos e pós-jogos em que houve derrotas.

Cada atleta reage diferentemente às situações vividas nas partidas, entretanto, sabe-se que atletas mais experientes tendem a reagir melhor diante de situações adversas. Torna- 
se importante identificar os estados de humor dos atletas que compõem a equipe para propor intervenções para regular as emoções. Nesse sentido, ao longo de uma competição os atletas vivem diferentes momentos (lesões, esgotamento físico, má distribuição dos intervalos entre as partidas) e conseguir identificar e controlar as emoções parece ser uma estratégia viável para melhorar o rendimento esportivo de uma equipe.

As variáveis que envolvem os estados de humor têm relação direta com as características e resultados dos esportes, não sendo diferente no futsal. Saber lidar com as emoções antes, durante e após as partidas, principalmente as emoções negativas, torna-se de suma importância para técnicos e membros da comissão técnica na busca pela continuidade de bons resultados. Futuras pesquisas devem evidenciar os estados de humor também em outras fases e em competições diferentes, comparando os resultados obtidos neste estudo, proporcionando assim novas perspectivas na intepretação dos dados e nas formas de intervenção junto aos atletas.

\section{REFERÊNCIAS}

Andrade, A., Bevilacqua, G. G., Coimbra, D. R., Pereira, F. S., \& Brandt, R. (2016). Sleep quality, mood and performance: A study of elite Brazilian volleyball athletes. Journal of Sports Science \& Medicine, 15(4), 601.

Araújo, S., \& Gomes, A. R. (2005). Efeitos de um programa de controle de ansiedade e de visualização mental na melhoria de competências psicológicas em jovens atletas: Uma intervenção na natação. Psicologia: Teoria, Investigação e Prática, 10(2), 211-225.

Beedie, C. J., Terry, P. C., \& Lane, A. M. (2000). The profile of mood states and athletic performance: Two meta-analyses. Journal of Applied Sport Psychology, 12(1), 49-68.

Brandt, R., Andrade, A., Segato, L., Kretzer, F. L., Díaz, J. D., \& Ramisa, A. V. (2010). Estados de ánimo de los deportistas de la selección brasileña de vela durante los juegos panamericanos. Revista de Psicología General y Aplicada: Revista de la Federación Española de Asociaciones de Psicología, 63(1), 75-85.

Brandt, R., Bevilacqua, G. G., \& Andrade A. (2017). Perceived sleep quality, mood states, and their relationship with performance among Brazilian elite athletes during a competitive period. Journal of Strength \& Conditioning Research, 31(4), 10331039 doi: 10.1519/JSC.0000000000001551.

Brandt, R., Viana, M. D. S., Segato, L., Kretzer, F. L., Carvalho, T. D., \& Andrade, A. (2011). Relações entre os estados de humor e o desempenho esportivo de velejadores de alto nível. Psicologia: Teoria e Prática, 13(1), 117-130.

Cratty, B. J. (1984). Psicologia no esporte. Rio de Janeiro: PrenticeHall do Brasil.

Cyrino, E. S., Altimari, L. R., Okano, A. H., \& de Faria Coelho, C. (2002). Efeitos do treinamento de futsal sobre a composição corporal e o desempenho motor de jovens atletas. Revista Brasileira de Ciência e Movimento, 10(1), 41-6.

de Rose Junior, D. (2002). A competição como fonte de estresse no esporte. Revista Brasileira de Ciência e Movimento, 10(4), 19-26.

Devonport, T. J., Lane, A. M., \& Hanin, Y. (2005). Emotional states of athletes prior to performance-induced injury. Journal of Sports Science \& Medicine, 4, 382-394

dos Santos, G. (2008). Relação dos estados transitórios de humor com a performance em competição de futebol de campo da cidade de Pouso Alegre/MG. Conexões, 6, 596-608. doi: 10.20396/conex.v6i0.8637860

Escobar, L., \& Lacerda, A. (2010). Identificação e caracterização dos estados de humor de atletas da seleção carioca de beach soccer durante o campeonato brasileiro. Movimento e Percepção, 11(16), 107-19.

Furley, P., Bertrams, A., Englert, C., \& Delphia, A. (2013). Ego depletion, attentional control, and decision making in sport. Psychology of Sport and Exercise, 14(6), 900-904.

Fullagar, H. H., Duffield, R., Skorski, S., Coutts, A. J., Julian, R., $\&$ Meyer, T. (2015). Sleep and recovery in team sport: Current sleep-related issues facing professional team-sport athletes. International Journal of Sports Physiology and Performance $10(8), 950-957$.

García, G. A. (2004). Caracterización de los esfuerzos en el fútbol sala basados en el estudio cinemático y fisiológico de la competición. Lecturas: Educación Física y Deportes, 77, 23.

Glazier, P. S. (2017). Towards a grand unified theory of sports performance. Human Movement Science, 56, 139-156.

Harrison, Y., \& Horne, J. A. (2000). The impact of sleep deprivation on decision making: A review. Journal of Experimental Psychology: Applied, 6(3), 236-249.

Lane, A. M., \& Terry, P. C. (2000). The nature of mood: Development of a conceptual model with a focus on depression. Journal of Applied Sport Psychology, 12(1), 16-33. doi: 10.1080/10413200008404211

Lane, A. M., Terry, P. C., Beedie, C. J., Curry, D. A., \& Clark, N. (2001). Mood and performance: Test of a conceptual model with a focus on depressed mood. Psychology of Sport and Exercise, 2(3), 157-172. doi:10.1016/S1469-0292(01)00007-3

Lazarus, R. S. (2000). How emotions influence performance in competitive sports. The Sport Psychologist, 14(3), 229-252.

Lowther, J., \& Lane, A. (2002). Relationships between mood, cohesion and satisfaction with performance among soccer players. Athletic Insight, 4(3), 57-69.

Rohlfs, I. C. P. M. (2006). Validação do teste de BRUMS para avaliação de humor em atletas e não atletas brasileiros (Dissertação de mestrado não publicada). Universidade do Estado de Santa Catarina, Florianópolis, SC.

Rohlfs, I. C. P. D. M., Rotta, T. M., Luft, C. D. B., Andrade, A., Krebs, R. J., \& Carvalho, T. D. (2008). A Escala de Humor de Brunel (Brums): Instrumento para detecção precoce da síndrome do excesso de treinamento. Revista Brasileira de Medicina do Esporte, 14(3), 176-181. doi: 10.1590/S151786922008000300003

Spielberger, C. D. (1991). Manual for the State-Trait AngerExpression Inventory. Odessa, FL: Psychological Assessment Resources.

Soares, B. H., \& Tourinho Filho, H. (2006). Análise da distância e intensidade dos deslocamentos, numa partida de futsal, nas diferentes posições de jogo. Revista Brasileira de Educação Física e Esporte, 20(2), 93-101. doi: 10.1590/S180755092006000200002

Souissi, N., Chtourou, H., Aloui, A., Hammouda, O., Dogui, M., Chaouachi, A., \& Chamari, K. (2013). Effects of timeof-day and partial sleep deprivation on short-term maximal performances of judo competitors. Journal of Strength and Conditioning Research 27, 2473-2480.

Terry, P. (1995). The efficacy of mood state profiling with elite performers: A review and synthesis. Sport Psychologist, 9, 309-309.

Terry, P. C., Lane, A. M., \& Fogarty, G. J. (2003). Construct validity of the Profile of Mood States - Adolescents for use 
with adults. Psychology of Sport and Exercise, 4(2), 125-139. doi:10.1016/S1469-0292(01)00035-8

Viana, M. F., Almeida, P., \& Santos, R. C. (2001). Adaptação portuguesa da versão reduzida do Perfil de Estados de HumorPOMS. Análise Psicológica, 19(1), 77-92. doi: 10.14417/ap.345

Vieira, L. F., Fernandes, S. L., Vieira, J. L. L., \& Vissoci, J. R. N. (2008). Estado de humor e desempenho motor: Um estudo com atletas de voleibol de alto rendimento. Revista Brasileira de Cineantropometria e Desempenho Humano, 10(1), 62-68.

Werneck, F. Z., Coelho, E. F., \& Ribeiro, L. C. (2002). Relação dos estados de humor e a performance em voleibolistas. In: M. Turin \& L. da Costa (Orgs.), Coletânea de textos em estudos olímpicos (pp. 337-350). Rio de Janeiro: Editora Gama Filho. 